\title{
From Fully-Packed Loops to Meanders: Exact Exponents
}

\section{P. Di Francesco,}

Service de Physique Théorique, C.E.A. Saclay,

F-91191 Gif sur Yvette, France

E-mail: philippe@spht.saclay.cea.fr

ABSTRACT: We address the meander problem "enumerate all topologically inequivalent configurations of a closed nonselfintersecting plane curve intersecting a given line through a fixed number of points". We show that meanders may be viewed as the configurations of a suitable fully-packed loop statistical model defined on a random surface. Using standard results relating critical singularities of a lattice model to its gravitational version on random surfaces, we predict the meander configuration exponent $\alpha=(29+\sqrt{145}) / 12$ and many other meandric exponents.

\section{Introduction}

In these notes we will be mainly dealing with the so-called meander problem, stated as follows: "Find the number $M_{n}$ of all topologically inequivalent configurations of a closed nonselfintersecting plane curve (road) intersecting a given line (river) through a fixed number $2 n$ of points (bridges)". This is an old problem: it can probably be traced back to some work by Poincaré (1911), and reemerged in various contexts since: as mathematical recreation [1], as folding problem [2] [3], in relation to the 16th Hilbert problem [4], in the theory of invariants of 3-manifolds [5], in computer science [6], in abstract algebraic terms [7] [8], and in its own right [9] [10].

Our main motivation is the study of the folding problem of polymer chains. Such a polymer is ideally described by a chain of identical line segments attached by their ends, which serve as hinges between adjacent segments. We will distinguish between closed and open polymers according to whether the chain forms a loop or is open with two free ends. We will be addressing the compact self-avoiding folding of such objects, namely study the various ways in which the polymer can be completely folded onto one of its segments.

To distinguish between the various ways of

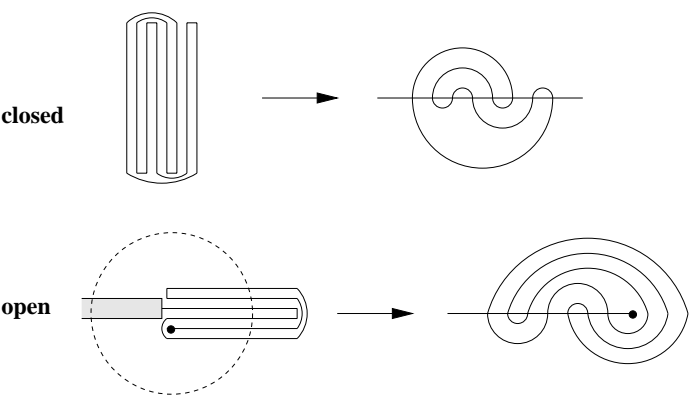

Figure 1: Two compactly folded polymers and the corresponding meanders. The first example is a closed polymer with 8 segments, and corresponds to a meander of order 8 . The second example is an open polymer with 6 segments, and corresponds to a semi-meander of order 7 .

compactly folding a closed polymer, we will represent the folded objects as meanderswith $2 n$ bridges. To visualize the relation between compactly folded closed polymers and meanders, it is simplest to imagine we draw a line perpendicular to the segments forming the folded polymer with a total of $2 n$ intersections (each segment intersects the line once), and then separate the various segments (see Fig.'.1.1.).

In the case of an open polymer with say $n-1$ segments, let us attach one of its ends to say a wall or a support (see Fig.'1-1.1), so as to prevent the 
polymer from winding around that end. Starting from a compactly folded configuration, let us again draw this time a circle that intersects each of the $n-1$ segments once, and also intersects the support once. Extending the polymer so as to let it form a half-line with its free end as origin, we form a planar configuration of a non-selfintersecting loop (road) crossing a halfline (river with a source) through $n$ points. These configurations considered up to smooth deformations preserving the topology are called semimeanders of order $n$. The total number of semimeanders of order $n$ is denoted by $\bar{M}_{n}$.

The approach developed in this note was first exposed in [11]: we will actually view meanders as configurations of a certain two-dimensional statistical model, formed by covering say the square lattice with fully-packed loops of two colors (one color for roads and one for rivers), but instead of defining the model on a fixed lattice, we define it on random surfaces of genus zero and fixed area, materialized by connected planar tetravalent graphs with fixed numbers of vertices. Meanders will be recovered by demanding that there be only one loop of each color in the relevant configurations.

Replacing the square lattice by a statistical sum over tetravalent graphs is a well-known process in physics: it amounts to the coupling of the critical two-dimensional statistical model at hand (here the fully packed two-color loop model) with two-dimensional quantum gravity. By noticing that our two-color fully-packed loop model on the square lattice is described in the continuum limit by a certain conformal theory, we will be able to use the known dictionary translating the scaling data of the conformal model into critical properties of its gravitational version, leading in particular to the exact singular behavior of the meander generating function, translated into the following meander number asymptotics

$$
M_{n} \sim C \frac{R^{2 n}}{n^{\alpha}} \quad \alpha=\frac{29+\sqrt{145}}{12}
$$

The number $\alpha$ is called the meander configuration exponent.

Similarly to (i. $\left(\begin{array}{l}1 \\ 1\end{array}\right)$, we will derive the following large $n$ asymptotics of the semi-meander num- bers

$$
\bar{M}_{n} \sim \bar{C} \frac{\bar{R}^{n}}{n^{\bar{\alpha}}}
$$

where it is expected that $\bar{R}=R[10]$ and the semi-meander configuration exponent reads [11]

$$
\bar{\alpha}=1+\frac{1}{24} \sqrt{11}(\sqrt{5}+\sqrt{29})
$$

\section{The fully-packed loop model for meanders}

\subsection{Fully-packed loop gas on the square lattice}
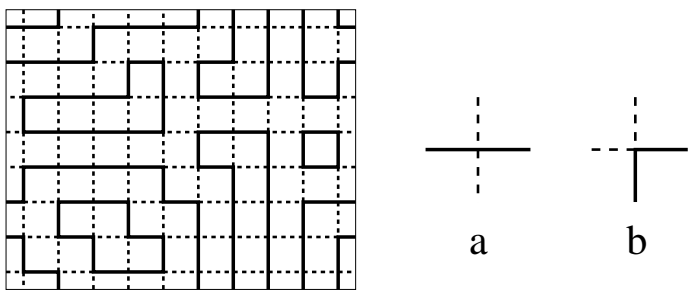

Figure 2: A typical Fully-Packed loop configuration on the square lattice. Assuming doubly periodic boundary conditions, there are 6 black loops (solid line) and 4 white ones (dashed lines). Up to rotations, the vertices of the model are of the two types (a) "crossing" or (b) "avoiding".

The fully-packed loop model's configurations are defined by assigning to each edge of the twodimensional square lattice either of two colors (say black or white, represented as solid or dashed lines in Fig. 2, also referred to as 1 and 2 in the following) in such a way that each vertex has exactly two black and two white incident edges. Up to obvious rotations, this gives rise to the only two vertex configurations depicted in Fig. 2 in which the black and white lines either avoid or cross each other. Note that with say periodic boundary conditions the black and white lines form loops on the lattice.

The fully-packed loop model is then further defined by assigning a weight $n_{1}$ per black loop and $n_{2}$ per white one, so that for instance the partition function reads

$$
Z_{\mathrm{FPL}}\left(n_{1}, n_{2}\right)=\sum_{\substack{\text { fully- packed loop } \\ \text { configurations }}} n_{1}^{L_{1}} n_{2}^{L_{2}},
$$

where $L_{i}$ denote the total numbers of loops of each color $i=1,2$. The model with partition 
function (2.1) is usually called the $\operatorname{FPL}^{2}\left(n_{1}, n_{2}\right)$ model.

The loop weights $n_{i}$ may be rephrased into local Boltzmann weights as follows. Let us assign to each black or white loop an arbitrary orientation, and attach to each vertex a local Boltzmann weight $e^{i \pi\left(\epsilon_{1} e_{1}+\epsilon_{2} e_{2}\right) / 4}$ where $\epsilon_{j}=1$ if the oriented loop of color $j$ makes a left turn, $\epsilon_{j}=0$ if it goes straight, and $\epsilon_{j}=-1$ if it makes a right turn. Summing over all possible orientations of all loops, we get a factor $2 \cos \pi e_{j}$ per loop of color $j$, therefore we reproduce the desired loop weights by setting $n_{1}=2 \cos \pi e_{1}$ and $n_{2}=2 \cos \pi e_{2}$.

\subsection{Conformal field theory description}

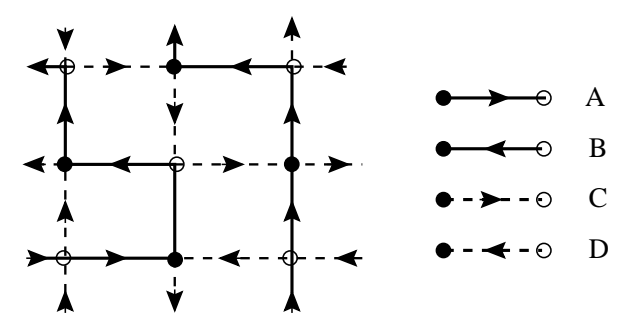

Figure 3: A typical configuration of the $\mathrm{FPL}^{2}$ model together with the bicoloration of its vertices (checkerboard of filled $(\bullet)$ and empty (०) dots). We have added the corresponding dictionary that allows to map the loop configurations onto $A, B, C, D$ labelings of the edges.

The $\operatorname{FPL}^{2}\left(n_{1}, n_{2}\right)$ model is known to be critical for $0 \leq n_{i} \leq 2$ [12], and is described in the continuum limit by a simple conformal field theory based on free scalar fields. To identify these, it is useful to rephrase the model as a (threedimensional) height model as follows. Starting from an oriented fully-packed black and white loop configuration, we first bicolor the vertices of the square lattice, say with alternating filled $(\bullet)$ and empty (o) dots. Then we use the dictionary of Fig. 3 to assign one of the four labels $A, B, C, D$ to each colored and oriented edge. With this convention, it is clear that edges of type $A B A B \ldots$ alternate along black loops, whereas edges of type $C D C D \ldots$ alternate along white loops, and that each vertex has one incident edge of each type $A, B, C, D$. It is clear that the fourlabeling with $A, B, C, D$ is in one-to-one corre- spondence with the coloring and orientation of edges of the FPL model. In particular, the orientation of a given black or white loop is reversed if we interchange the $A$ and $B$ or $C$ and $D$ labels along the loop.

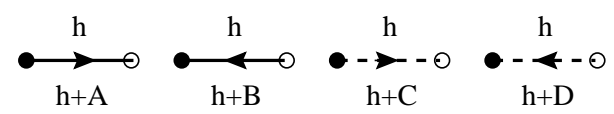

Figure 4: Rules determining the change of the height variable across labeled edges. We adopt the Ampère convention that the height is increased (resp. decreased) by the edge value if the arrow of the edge points to the left (resp. right). The edge labels must be interpreted as three-dimensional vectors with the respective values $\mathbf{A},-\mathbf{B}, \mathbf{C},-\mathbf{D}$

The above colors allow for defining a dual vector height variable on the center of each face of the lattice. Indeed, viewing as vectors the $A, B, C, D$ labeling of the edges of the lattice, let us arbitrarily fix the height to be zero on a given face of the lattice, and define it on all faces by successive use the rules of Fig. 4 for the transition from a face to any of its neighbors. Note that it is necessary to impose the condition $\mathbf{A}+\mathbf{B}+\mathbf{C}+\mathbf{D}=0$ to ensure that the heights are consistently defined around each vertex. We may therefore assume in all generality that $\mathbf{A}, \mathbf{B}, \mathbf{C}, \mathbf{D}$ are actually four vectors in $\mathbb{R}^{3}$ with vanishing sum; let us take for definiteness $\mathbf{A}, \mathbf{B}, \mathbf{C}, \mathbf{D}$ to be the four unit vectors pointing from the center of a tetrahedron towards its vertices. The heights are then clearly three-dimensional, as linear combinations of $\mathbf{A}, \mathbf{B}, \mathbf{C}, \mathbf{D}$. In the continuum limit, it was argued that the three-dimensional height variable turns into a three-dimensional scalar field. Moreover the symmetries of the model completely fix the form of the action for these fields and the corresponding field theory is conformal, with central charge $c_{\mathrm{FPL}}\left(n_{1}, n_{2}\right)=3-6\left(\frac{e_{1}^{2}}{1-e_{1}}+\frac{e_{2}^{2}}{1-e_{2}}\right)$, with $e_{j}$ as above.

\section{From fully-packed loop gas to me- anders}

\subsection{Meanders: the coupling to gravity}

To finally get to meanders, we must consider the coupling of the $\operatorname{FPL}^{2}\left(n_{1}, n_{2}\right)$ model to twodimensional quantum gravity, by allowing the 
square lattice to fluctuate into arbitrary fourvalent graphs. Then for each such graph the fully-packed loop model is still defined by coloring the edges black or white and allowing only the vertices $\mathrm{a}$ and $\mathrm{b}$ of Fig. 2. Each colored loop is then weighted by the appropriate $n_{j}$ factor $j=1,2$.

If we try to go through the steps of the previous section, namely to transform the model into a height model, we stumble on the issue of bicolorability of the vertices on the random fourvalent graphs. Indeed, not all graphs are vertexbicolorable. So the coupling to gravity stricto sensu (sum over arbitrary four-valent graphs) will destroy this property and affect the degrees of freedom of the model.

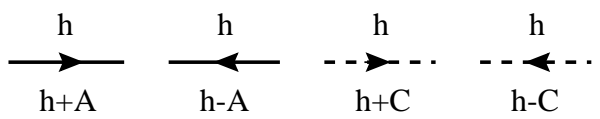

Figure 5: Rules determining the change of the height variable across labeled edges in the nonbicolored case. These rules are identical to those of Fig. 4, with the further restriction that $\mathbf{B}=-\mathbf{A}$ and $\mathbf{D}=-\mathbf{C}$, allowing to ignore the bicoloration of vertices.

Indeed, having lost the bicolorability of vertices, it is no longer possible to distinguish between $A$ and $B$ labels on one hand, and $C$ and $D$ on the other. We may still define an edge-labeling of the graph in one-to-one correspondence with oriented colored fully-packed loop configurations on the graph, but with vectors $\mathbf{A}, \mathbf{B}, \mathbf{C}, \mathbf{D}$. satisfying the two constraints $\mathbf{A}+\mathbf{B}=0$ and $\mathbf{C}+\mathbf{D}=0$, and picking say $\mathbf{A}$ and $\mathbf{C}$ to be two perpendicular unit vectors in $\mathbb{R}^{2}$. The correspondence between color/orientation and $A, C$ labels reads as in Fig. 5. As in Eq. (2.1), the model is further completed by attaching weights $n_{j}$ to each loop of color $j=1,2$. We may now define a height variable on the centers of the faces of the graph, by use of the previous rules. The main difference is that the height now lives in two dimensions (the plane generated by $\mathbf{A}$ and $\mathbf{C}$ ). This "dimensional reduction" results in a reduction $c \rightarrow c-1$ of the central charge of the underlying conformal theory, namely

$$
c\left(n_{1}, n_{2}\right)=2-6\left(\frac{e_{1}^{2}}{1-e_{1}}+\frac{e_{2}^{2}}{1-e_{2}}\right)
$$

The partition function of the fully-packed model coupled to quantum gravity, referred to as the $\operatorname{GFPL}^{2}\left(n_{1}, n_{2}\right)$ model, reads in genus zero

$$
\begin{array}{r}
Z_{\mathrm{GFPL}}\left(n_{1}, n_{2} ; x, y\right)=\sum_{\substack{\text { four- valent planar } \\
\text { graphs }}} \frac{1}{|\operatorname{Aut}(\Gamma)|} \times \\
\times \sum_{\substack{\mathrm{FPL} \text { configs. } \\
\text { on } \Gamma}} n_{1}^{L_{1}} n_{2}^{L_{2}} x^{V_{a}(\Gamma)} y^{V_{b}(\Gamma)}(3.2)
\end{array}
$$

where the sum extends over all the genus zero four-valent graphs $\Gamma$, and $|\operatorname{Aut}(\Gamma)|$ is the order of the symmetry group of $\Gamma$, while we have also denoted by $V_{a}, V_{b}$ the total numbers of vertices of type $\mathrm{a}$ and $\mathrm{b}$ defined in Fig. 2 in the particular loop configuration, namely we have weighted each crossing of a black and a white loop by $x$ and each avoiding by $y$. When $x=y$, these are interpreted as the cosmological constant, as the total number of vertices $V_{a}+V_{b}$ of $\Gamma$ is also the area of the corresponding dual random surface.

To generate meanders, we must now extract from (3.2) the configurations with only one black and one white loop, that will respectively play the role of the river and the road. This is done by taking the limit $n_{1}, n_{2} \rightarrow 0$ in (3.2), resulting in

$$
\begin{gathered}
Z_{\mathrm{GFPL}}(x, y)=\left.\frac{Z_{\mathrm{GPPL}}\left(n_{1}, n_{2} ; x, y\right)-1}{n_{1} n_{2}}\right|_{n_{i}=0} \\
=\sum_{\substack{n, p \geq 0 \\
n+p \geq 1}} \frac{x^{2 n} y^{p}}{2(2 n+p)} \mu_{2 n, p}
\end{gathered}
$$

where we have denoted by $\mu_{2 n, p}$ the total number of tangent meanders with $2 n$ crossings and $p$ tangency points, i.e. configurations of a nonselfintersecting circuit (road) crossing a line (river) through $2 n$ points (bridges=a-type vertices) and touching the river $p$ times (tangent contacts $=\mathrm{b}-$ type vertices). The usual meander numbers defined in [3] correspond to only crossings and no tangent points and read $M_{n}=\mu_{2 n, 0}$.

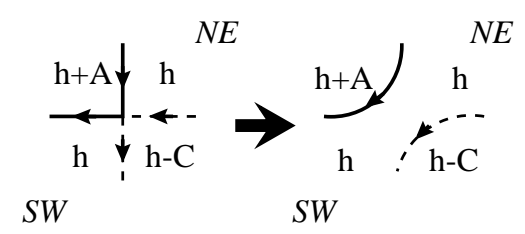

Figure 6: A type b vertex of the $\mathrm{FPL}^{2}$ gravitational model, together with its dual height configuration. We note that the NE and SW heights are identical. We may therefore undo the vertex as shown. 
The meanders are therefore generated by the function (3.3) for $y=0$. Let us now show that the universality class of the tangent meanders is the same as that of meanders. In the transformation into a (two-dimensional) height model, the "tangency" vertex b of Fig. 2 corresponds to the arrangements of heights on the adjacent faces of Fig. 6. We notice that in the case of the "tangency" vertex b, the NE and SW heights are identical, irrespectively of the orientations of the two loops. This means that as far as the height variable is concerned this vertex may be simply removed as shown. We conclude that the b vertex of the model is irrelevant, i.e. the parameter $y$ does not drive the model out of the universality class of meanders.

As a consequence, we expect the following asymptotics for $N=2 n+p$ large of the partition function $\mu_{N}(x, y)$ for tangent meanders with a total $N$ of bridges and tangency points:

$\mu_{N}(x, y)=\sum_{\substack{n, p>0 \\ 2 n+p=N}} x^{2 n} y^{p} \mu_{2 n, p} \sim C(x, y) \frac{R(x, y)^{N}}{N^{\alpha}}$

where the configuration exponent $\alpha$ is independent of $x$ and $y$ for $x>0$ and $y \geq 0$. In particular, when $y=0, \alpha$ is identified with the meander configuration exponent (11.iיi).

To conclude this section, thus far we have determined that the universality class of meanders is that of a conformal field theory coupled to quantum gravity, with central charge $(\overline{3} .1 \overline{1})$ at $n_{1}=n_{2}=0$, namely $c(0,0)=-4$.

\subsection{Field theory description of meandric numbers}

The coupling of a conformal field theory with central charge $c \leq 1$ to two-dimensional quantum gravity has a simple field-theoretical description in terms of the Liouville field describing the conformal classes of metrics of the surfaces. This has led to a number of results, including the precise determination of various critical exponents. Indeed, the gravitational theory (say on genus zero surfaces) displays a critical behavior as a function of the cosmological constant $x$. In particular, there exists a finite value $x_{c}$ of $x$ at which the (connected) partition function behaves as $Z(x) \sim$ $\left(x_{c}-x\right)^{2-\gamma(c)}$ where the string susceptibility exponent $\gamma$ is related to the central charge $c$ through [13]

$$
\gamma(c)=\frac{c-1-\sqrt{(1-c)(25-c)}}{12}
$$

When applied to the $\operatorname{GFPL}^{2}(0,0)$ model of the previous section (with $c=-4$ ), we find that

$$
\gamma \equiv \gamma(-4)=-\frac{5+\sqrt{145}}{12}
$$

Applying this to the series expansion of the partition function $Z_{\mathrm{GFPL}}(x)=\sum_{n>1} \frac{x^{2 n}}{4 n} M_{n}$, we deduce the asymptotic behavior

$$
M_{n} \sim C \frac{x_{c}^{-2 n}}{n^{\alpha}} \quad \alpha=2-\gamma=\frac{29+\sqrt{145}}{12}
$$

in agreement with (11) with $R=1 / x_{c}$.

In addition, a number of the operators of the flat space conformal theory get dressed by gravity, in such a way that they acquire anomalous scaling dimensions. Any given operator $\phi_{k}$ with conformal dimensions $h_{k}=\bar{h}_{k}$, is dressed into an operator $\tilde{\phi}_{k}$ with dressed dimension $\Delta_{k}$ such that the correlation functions behave as

$$
\left\langle\tilde{\phi}_{k_{1}} \tilde{\phi}_{k_{2}} \ldots \tilde{\phi}_{k_{p}}\right\rangle \sim\left(x_{c}-x\right)^{2-\gamma+\Sigma\left(\Delta_{k_{i}}-1\right)}
$$

when $x$ approaches the critical value $x_{c}$, and where the dressed dimension $\Delta_{k}$ is related to the flat space conformal dimension $h_{k}$ through

$$
\Delta_{k}=\frac{\sqrt{1-c+24 h_{k}}-\sqrt{1-c}}{\sqrt{25-c}-\sqrt{1-c}}
$$

Let us expose the operator content of the $c=-4$ conformal theory describing the flat space version of our model. For generic values of $n_{1}, n_{2}$, it has a continuum description as a two-component scalar field with charges at infinity. More precisely, it is a Coulomb gas made of two decoupled scalar fields, with $c=c\left(n_{i}\right)=1-6 e_{i}^{2} /\left(1-e_{i}\right)=$ -2 at $n_{i}=0\left(e_{i}=1 / 2\right)$ respectively, each viewed as the effective field theory of loops of one color. In particular, within each scalar field theory (indexed by the color $i=1,2$ ), there exist operators $\psi_{k}^{(i)}(z)$ that create $k$ oriented defect lines (of color i) for the scalar field, with conformal dimensions

$$
h_{k}^{(i)}=\frac{k^{2}-4}{32}
$$


at $n_{i}=0$. In the Coulomb gas formalism, these correspond to electromagnetic operators with electric charge $e_{i}$ (spin-wave) and magnetic charge $\pm k / 2$ (vortex), according to whether the defect line is oriented from or to the insertion point, and $k$ is a strictly positive integer. For $k=0$ Eqn. (B. $\overline{1} \overline{1}$ ) must be replaced by $h_{0}^{(i)}=0$ corresponding to the identity operator. The correlation functions must have a vanishing total magnetic charge.

Let us now study the dressing of these operators by gravity, and interpret them in meandric terms. The dressed operator $\tilde{\psi}_{k}^{(i)}$ again corresponds to the creation of a vertex with $|k|$ outcoming $(k>0)$ or incoming $(k<0)$ lines of color $i$, and the corresponding dressed dimension (3.8i)$(3.9)$ reads $\Delta_{k}^{(i)}=\left(\frac{1}{2} \sqrt{8+3 k^{2}}-\sqrt{5}\right) /(\sqrt{29}-$ $\sqrt{5})$. The main application concerns the two point function describing the insertion of a segment of river (color 1 )

$$
\left\langle\tilde{\psi}_{1}^{(1)} \tilde{\psi}_{-1}^{(1)}\right\rangle \sim\left(x_{c}-x\right)^{2 \Delta_{1}-\gamma}
$$

Recall that when we take the limit $n_{1}, n_{2} \rightarrow$ 0 , only diagrams with one connected component of river and one of road are selected. In the case of $(\overline{3} . \overline{1} \overline{1})$, the river forms a segment, around which the road can freely wind. To fix ambiguities, let us send one end of the river to infinity (say to the left) and therefore represent the river as a half-line as in Fig. I'. The number of configurations of a closed road crossing a half-line (river with a source) through $n$ bridges is defined as the semi-meander number $\bar{M}_{n}$. We immedi-

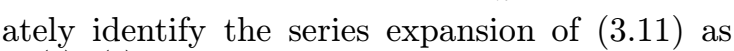
$\left\langle\tilde{\psi}_{1}^{(1)} \tilde{\psi}_{-1}^{(1)}\right\rangle=\sum_{n \geq 1} \bar{M}_{n} x^{n}$. We therefore deduce the semi-meander asymptotics

$\bar{M}_{n} \sim \bar{c} \frac{x_{c}{ }^{-n}}{n^{\bar{\alpha}}}, \bar{\alpha}=1+2 \Delta_{1}-\gamma=1+\frac{\sqrt{11}}{24}(\sqrt{5}+\sqrt{29})$

In [11], a number of other results have been presented, all corresponding to more sophisticated river geometries, and making use of the magnetic defect operators $\tilde{\psi}_{k}^{(i)}$, that create river vertices with $|k|$ branches.

Acknowledgements : This research is supported in part by European TMR contract ERBFMRXCT960012.

\section{References}

[1] A. Sainte-Laguë, Avec des nombres et des lignes (Récréations Mathématiques), Vuibert, Paris (1937).

[2] J. Touchard, Contributions à l'étude du problème des timbres poste, Canad. J. Math. 2 (1950) 385-398; W. Lunnon, A map-folding problem, Math. of Computation 22 (1968) 193199.

[3] P. Di Francesco, O. Golinelli and E. Guitter, Meander, folding and arch statistics, Mathl. Comput. Modelling 26 (1997) 97-147.

[4] V. Arnold, The branched covering of $\mathrm{CP}_{2} \rightarrow \mathrm{S}_{4}$, hyperbolicity and projective topology, Siberian Math. Jour. 29 (1988) 717-726.

[5] K.H. Ko, L. Smolinsky, A combinatorial matrix in 3-manifold theory, Pacific. J. Math 149 (1991) 319-336.

[6] K. Hoffman, K. Mehlhorn, P. Rosenstiehl and R. Tarjan, Sorting Jordan sequences in linear time using level-linked search trees, Information and Control 68 (1986) 170-184.

[7] P. Di Francesco, O. Golinelli and E. Guitter, Meanders and the Temperley-Lieb Algebra, Commun. Math. Phys. 186 (1997), 1-59.

[8] R. Bacher, Meander Algebras, prépublication de l'Institut Fourier n ${ }^{\circ} 478$ (1999).

[9] S. Lando and A. Zvonkin, Plane and Projective Meanders, Theor. Comp. Science 117 (1993) 227-241, and Meanders, Selecta Math. Sov. 11 (1992) 117-144.

[10] P. Di Francesco, O. .Golinelli and E. Guitter, Meanders: a direct enumeration approach, Nuc. Phys. B 482 [FS] (1996) 497-535.

[11] P. Di Francesco, O. Golinelli and E. Guitter, Meanders: exact asymptotics, Nucl.Phys. B570 (2000) 699-712.

[12] J. Jacobsen and J. Kondev, Field theory of compact polymers on the square lattice, Nucl. Phys. B 532 [FS], (1998) 635-688, Transition from the compact to the dense phase of two-dimensional polymers, J. Stat. Phys. 96, (1999) 21-48.

[13] V.G. Knizhnik, A.M. Polyakov and A.B. Zamolodchikov, Mod. Phys. Lett. A3 (1988) 819; F. David, Mod. Phys. Lett. A3 (1988) 1651; J. Distler and H. Kawai, Nucl. Phys. B321 (1989) 509. 